\title{
Meta-Analysis of Ventilated versus Spontaneously Breathing Patients in Predicting Fluid Responsiveness by Inferior Vena Cava Variation
}

\author{
Xiang $\mathrm{Si}^{1}$, Daiyin $\mathrm{Cao}^{2}$, Hailin $\mathrm{Xu}^{3}$, Xiangdong Guan ${ }^{*}$ \\ ${ }^{1}$ Department of Surgical Intensive Care Unit, The First Affiliated Hospital of Sun Yat-sen University, Guangzhou, China \\ ${ }^{2}$ Department of Critical Care Medicine, The Sixth Affiliated Hospital of Sun Yat-sen University, Guangzhou, China \\ ${ }^{3}$ Transplantation Department, The Second Affiliated Hospital of Guangzhou Medical University, Guangzhou, China \\ Email: james_sixiang@163.com, manfeng2005@sina.com, xuhlin3@icloud.com, ‘xiangdguan@126.com
}

How to cite this paper: $\mathrm{Si}, \mathrm{X}$., Cao, D.Y., Xu, H.L. and Guan, X.D. (2018) Meta-Analysis of Ventilated versus Spontaneously Breathing Patients in Predicting Fluid Responsiveness by Inferior Vena Cava Variation. International Journal of Clinical Medicine, 9, 760-777. https://doi.org/10.4236/ijcm.2018.910063

Received: October 10, 2018

Accepted: October 23, 2018

Published: October 26, 2018

Copyright (c) 2018 by authors and Scientific Research Publishing Inc. This work is licensed under the Creative Commons Attribution International License (CC BY 4.0).

http://creativecommons.org/licenses/by/4.0/

\begin{abstract}
Purpose: Respiratory variation in inferior vena cava $(\Delta \mathrm{IVC})$ has been extensively studied in predicting fluid responsiveness, but the results are conflicting. We performed a systemic review and meta-analysis of studies aiming at investigating the diagnostic accuracy of $\triangle \mathrm{IVC}$ in predicting fluid responsiveness. Methods: MEDLINE, EMBASE, Cochrane Database and Web of Science were screened for relevant original and review articles from inception to July 2016. The meta-analysis determined the pooled sensitivity, specificity, diagnostic odds ratio (DOR) and area under the ROC curve (AUROC). In addition, subgroup analyses were performed in mechanically ventilated patients and spontaneously breathing patients. Results: A total of 20 studies involving 635 patients were included. Cutoff values of $\triangle \mathrm{IVC}$ varied from $12 \%$ to $42 \%$, the pooled sensitivity and specificity was $0.68(0.62-0.75)$ and $0.80(0.75-$ $0.85)$, respectively. The DOR was $14.2(6.0$ - 33.6) and the AUROC was 0.86 (0.78 - 0.93). Subgroup analysis showed better diagnostic performance in patients on mechanical ventilation than in spontaneously breathing patients with higher sensitivity ( 0.75 vs. 0.56 ), specificity ( 0.82 vs. 0.78$)$, DOR ( 22.9 vs. 7.9) and AUROC ( 0.90 vs. 0.80 ). The best threshold of $\triangle \mathrm{IVC}$ in patients on mechanical ventilation was IVC distensibility index $(\triangle \mathrm{dIVC} \geq 17 \% \pm 4 \%)$, compared to IVC collapsibility index ( $\triangle$ cIVC $\geq 33 \% \pm 12 \%$ ) in spontaneously breathing patients. Conclusion: $\triangle \mathrm{IVC}$ is not an accurate predictor of fluid responsiveness in patients with acute circulatory failure. In patients on mechanical ventilation, the predicting ability of $\triangle \mathrm{IVC}$ was moderate with acceptable sensitivity and specificity; in spontaneously breathing patients, the specificity remains acceptable but its sensitivity is poor.
\end{abstract}




\section{Keywords}

Fluid, Responsiveness, Inferior Vena Cava Variation

\section{Introduction}

Hypovolemia is a very frequent clinical situation in the intensive care unit (ICU) and is primarily treated with volume expansion (VE). The only goal of VE is to improve the cardiac output (CO) of the patients especially those with acute circulatory failure [1]. However, multiple studies have demonstrated that only approximately $50 \%$ of hemodynamically unstable patients respond to VE in the ICU [2]. It is therefore essential to have reliable tools to predict the efficacy of VE and ultimately distinguish patients who may benefit from VE from those who are unlikely to respond. Recently, many studies have focused on the prediction of fluid responsiveness. Static hemodynamic indices have been of little value in predicting fluid responsiveness [3] [4]. In contrast, dynamic indices, based on analysis of preload dependence, have been validated as factors that can help predict fluid responsiveness [3] [5] [6] [7]. However, because of invasiveness and high cost, the application of these indices is of limited use in emergency rooms and general wards.

Bedside point-of-care ultrasonography has gained considerable attention because of noninvasiveness, rapid diagnosis and low cost [8]. The diameter of the inferior vena cava (IVC) is easily recorded by transthoracic echocardiography (TTE) in a subcostal view. Because of the heart-lung interactions, the maximum IVC diameter (IVCmax) and minimum IVC diameter (IVCmin) can be measured during thecycle of breath. Then, a term named respiratory variation in IVC diameter $(\triangle \mathrm{IVC})$ can be calculated. In recent years, intensivist had increasing interesting on $\triangle \mathrm{IVC}$ for predicting fluid responsiveness.

Following the first study demonstrating the accuracy of the $\triangle I V C$, it has been extensively investigated for its usefulness. In 2014, a meta-analysis pooling eight studies published at that time confirmed that $\triangle \mathrm{IVC}$ is of great value in predicting fluid responsiveness [9]. However, since this meta-analysis, conflicting findings on its accuracy have been reported in a number of publications.

In order to clarify these mixed results and assess the ability of $\triangle \mathrm{IVC}$ to predict fluid responsiveness, we conducted a systemic review of all these studies and performed a meta-analysis, with hypothesis that $\triangle I V C$ performs well in predicting fluid responsiveness.

\section{Materials and Methods}

\subsection{Clinical Research Question}

The clinical research question was: What is the sensitivity and specificity of the $\triangle \mathrm{IVC}$ when using it to predict fluid responsiveness? 


\subsection{PICO Statement [10]}

The PICO statement is as the following:

P-patient, problem or population: patients with acute circulatory failure in whom the effect of volume expansion (VE) is unknown and needs to be predicted.

I-intervention: Inferior vena cava (IVC) diameter was examined subcostally and measured in M-mode or 2D mode, $2 \mathrm{~cm}$ before the IVC joined the right atrium. The IVC respiratory variation $(\triangle I V C)$ was calculate by recordingthe largest and smallest IVCdiameter at end-inspiration or end-expiration.

C-comparison, control, and comparator: Fluid responsiveness was defined as a significant increase of stroke volume (SV), cardiac output (CO) or other surrogates during a $\mathrm{VE}$.

O-outcomes: Ability of the $\triangle \mathrm{IVC}$ to predict fluid responsiveness.

\subsection{Searching Strategy, Study Identification and Data Extraction}

Our aim was to identify all studies evaluating the ability of the $\triangle \mathrm{IVC}$ to predict fluid responsiveness compared to the increase in SV, CO or other surrogates induced by subsequent VE.

We searched the MEDLINE, EMBASE, Cochrane and Web of Science databases for relative studies published in English from inception to July 2016. The key words we used consist of term related to IVC ("inferior vena cava", "caval index", "collapsibility" and "distensibility") and terms related to volume status ("fluid or volume or preload responsiveness", "fluid or preload challenge", "preload dependence or independence or dependency or independency", "functional haemodynamic monitoring" and "fluid therapy or management"). These key words were searched separately by two groups using different combination strategy. We also looked for relevant articles cited in review articles, commentaries and editorials. The search was performed repeatedly until no new studies could be found.

Study identification was performed in two steps. Step 1 comprised screening for titles and abstracts, and step 2, review of full texts of studies obtained in step 1. We only included studies investigating the accuracy of the $\triangle I V C$ that were published in full text or accepted for publication in indexed journals. Excluded criteria were 1) studies using central venous pressure or right atrial pressure as the reference standard, because these static parameters cannot predict fluid responsiveness accurately; 2) studies measuring IVC with techniques other than ultrasonography; 3) studies involving animals and healthy volunteers. Two reviewers process searching independently, disagreement was settled by a third opinion. The quality of the included studies was evaluated by using the QUADAS-2 scale [11]. The meta-analysis was performed according to the PRISMA statement.

Important information was extracted from the included articles using a standardized data form by two reviewers. Extracted data include the name of the first 
author, publication year, characteristics of the investigated population, sample size, respiratory pattern, the device for IVC measurement, formula for the calculation of $\triangle \mathrm{IVC}$, definition of fluid responsiveness and volume challenge strategy, the number of true positives, true negatives, false positives and false negatives, sensitivity, specificity, the area under the receiver operation characteristics curve (AUROC) and the best threshold of $\triangle \mathrm{IVC}$ which is used to predict the fluid responsiveness.

\subsection{QUADAS-2 Quality Assessment in Included Studies}

Included studies were assessed for their quality based on the Quality Assessment of Diagnostic Accuracy Studies-2 (QUADAS-2) protocol. QUADAS-2 scale [11] was made up of 4 domains: patient selection, index test, reference standard, and flow and timing. Each domain is assessed in terms of risk of bias, and the first 3 domains are also assessed in terms of concerns regarding applicability. For the "patient selection" domain, we examined whether patients were consecutively included and whether inappropriate exclusions were avoided. For the "index test" domain, we examined whether the threshold used to define volume responsiveness was pre-specified. For the "reference standard" domain, we examined whether the result of VE on SV, CO or surrogates was assessed without knowledge of $\triangle \mathrm{IVC}$ result. Finally, for the "flow and timing" domain, the authors examined whether there was an appropriate interval between IVC measurement and VE, whether patients received the same VE and whether all patients were included in the analysis. For each criterion, the risk was judged as high, low and unclear.

\subsection{Statistical Analysis}

We performed a meta-analysis in order to determine the pool sensitivity, specificity and diagnostic odds ratio (DOR). In addition, the pooled area under the ROC curve (AUROC) and threshold for $\triangle I V C$ as a predictor of fluid responsiveness was also evaluated. To investigate a threshold effect, we calculated the Spearman correlation coefficient between sensitivity and specificity. Homogeneity between studies was tested by the Chi squared test and $P$ index. According to heterogeneity, we adopted a random effect model by using the method of DerSimonian-Laird from the Mantel-Haenszel model. We compared studies with ICU setting versus non-ICU setting making the hypothesis that $\triangle \mathrm{IVC}$ could be more reliable in ICU patients. We compared studies with adults versus children making the hypothesis that $\triangle \mathrm{IVC}$ could be more reliable in adults. We compared studies with different devices for measuring IVC making the hypothesis that one device is better than the others. We compared studies with three different formulas for the calculation of $\triangle \mathrm{IVC}$ making the hypothesis that one formula is better than the others. We compared studies with patients on mechanical ventilation versus studies with spontaneously breathing patients, testing the hypothesis that the reliability of $\triangle \mathrm{IVC}$ is better in patients on mechanical ventilation. We compared studies where fluid responsiveness was defined by an increase in SV, 
CO or surrogate $\geq 15 \%$ versus studies with other definitions of fluid responsiveness, testing the hypothesis that the reliability of $\triangle \mathrm{IVC}$ is better when fluid responsiveness is defined by a larger increase. We compared studies where SV, CO or surrogate were measured by echocardiography versus studies where they were measured by other methods, testing the hypothesis that the reliability of $\triangle \mathrm{IVC}$ is better when SV, CO or surrogate were measured by echocardiography. Finally, we compared studies where VE was performed with versus studies where it was performed by colloids, testing the hypothesis that the reliability of $\triangle \mathrm{IVC}$ is better when VE is performed with colloids. Causes of heterogeneity were also investigated by meta-regression based on the Littenberg and Mose linear model.

Results are expressed as mean (95\% confidence interval) or as mean \pm standard deviation. The meta analysis was performed with Meta-Disc v.1.4 (Universidad Complutense, Madrid, Spain). The additional statistical analysis was performed with MedCal 15.2.2 (MedCal Software, Mariakerke, Belgium). A two-tailed $\mathrm{p}<0.05$ was considered to statistical significance.

\section{Results}

\subsection{Characteristics of Included Studies}

A flow chart of the study selection is provided in Figure 1. Our initial search identified 399 citations. 379 of them were excluded: 320 for not relating to the subject, 49 for being reviews, letters, guidelines, case reports and editorials, 3 for not writing in English, 4 for not using proper reference standard, 3 for being animal experiments. Finally, a total of 20 studies [12]-[31] reported the ability of $\triangle I V C$ to predict fluid responsiveness were included in our analysis.

Characteristics of included studies are listed in Table 1. Sample sizes were

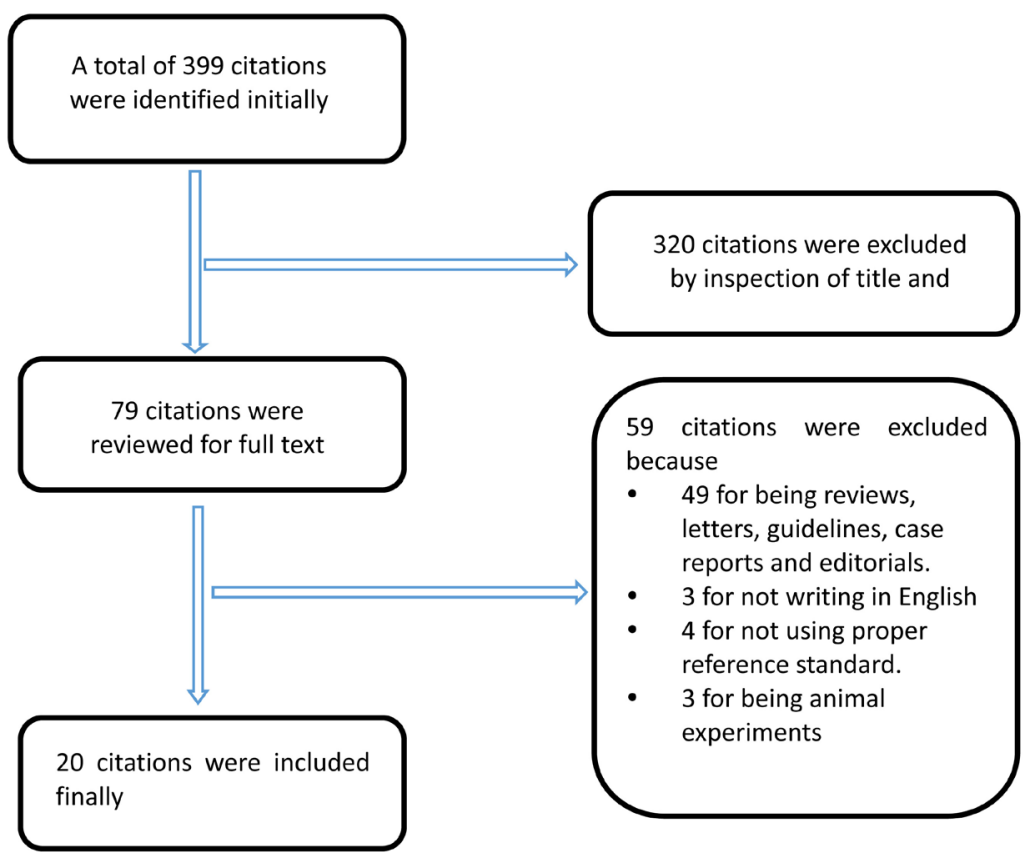

Figure 1. Flow chart of study selection. 
Table 1. Characteristics of the studies included.

\begin{tabular}{|c|c|c|c|c|c|c|c|c|c|}
\hline Study & $\begin{array}{l}\text { Type of } \\
\text { patients }\end{array}$ & $\begin{array}{l}\text { Sample } \\
\text { size }\end{array}$ & Setting & Type of device & $\begin{array}{l}\text { Method for } \\
\text { reference } \\
\text { standard }\end{array}$ & $\begin{array}{l}\text { Respiratory } \\
\text { pattern }\end{array}$ & Index formula & $\begin{array}{l}\text { reference } \\
\text { standard }\end{array}$ & $\begin{array}{l}\text { volume } \\
\text { expansion }\end{array}$ \\
\hline $\begin{array}{c}\text { Barbier et al. } 2004 \\
\text { [12] }\end{array}$ & adults & 20 & ICU & Philips & 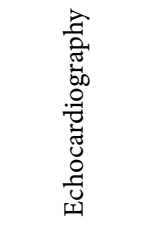 & $\begin{array}{c}\text { Mechanical } \\
\text { ventilation }(\mathrm{TV}=8.5 \\
\pm 1.5 \mathrm{~mL} / \mathrm{kg} ; \mathrm{PEEP}= \\
\left.4 \pm 2 \mathrm{~cm} \mathrm{H} \mathrm{H}_{2} \mathrm{O}\right)\end{array}$ & $\begin{array}{c}(\mathrm{IVCmax}- \\
\text { IVCmin)/IVCmin }\end{array}$ & CI > 15\% & $\begin{array}{l}7 \mathrm{ml} / \mathrm{kg} \\
\text { plasma }\end{array}$ \\
\hline $\begin{array}{c}\text { Feissel et al. } 2004 \\
\text { [13] }\end{array}$ & adults & 39 & ICU & Not mention & 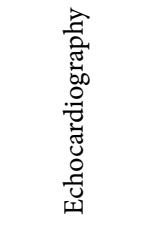 & $\begin{array}{c}\text { Mechanical } \\
\text { ventilation } \\
(\mathrm{TV}=8-10 \mathrm{~mL} / \mathrm{kg})\end{array}$ & $\begin{array}{c}(\text { IVCmax - } \\
\text { IVCmin)/[(IVCmax } \\
+ \text { IVCmin)/2] }\end{array}$ & $\mathrm{CO}>15 \%$ & 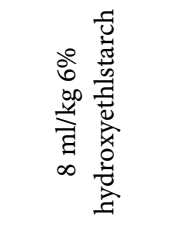 \\
\hline $\begin{array}{l}\text { Moretti and Pizzi } \\
\quad 2010[14]\end{array}$ & adults & 29 & ICU & $\begin{array}{c}\text { Esaote MyLab } 30 \\
\text { CV }\end{array}$ & 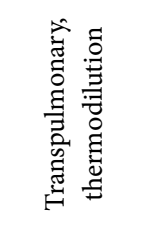 & $\begin{array}{c}\text { Mechanical } \\
\text { ventilation }(\mathrm{TV}=8 \\
\mathrm{mL} / \mathrm{kg} ; \mathrm{PEEP}=0 \mathrm{~cm} \\
\left.\mathrm{H}_{2} \mathrm{O}\right)\end{array}$ & $\begin{array}{c}(\mathrm{IVCmax}- \\
\text { IVCmin)/IVCmin }\end{array}$ & $\mathrm{CI}>15 \%$ & 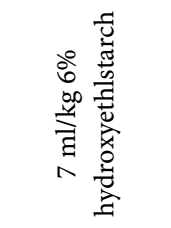 \\
\hline $\begin{array}{c}\text { Deok et al. } 2010 \\
{[15]}\end{array}$ & children & 21 & Pediatrics & $\begin{array}{l}\text { Acuson Cypress } \\
\text { Diagnostic } \\
\text { Ultrasound } \\
\text { System }\end{array}$ & 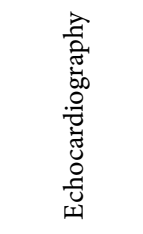 & $\begin{array}{c}\text { Mechanical } \\
\text { ventilation }(\mathrm{TV}=10 \\
\mathrm{mL} / \mathrm{kg} ; \mathrm{PEEP}=0 \mathrm{~cm} \\
\left.\mathrm{H}_{2} \mathrm{O}\right)\end{array}$ & $\begin{array}{c}(\text { IVCmax - } \\
\text { IVCmin)/[(IVCmax } \\
+ \text { IVCmin)/2] }\end{array}$ & $\mathrm{SV}>15 \%$ & 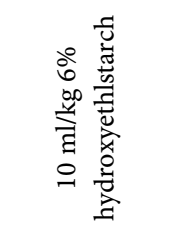 \\
\hline $\begin{array}{c}\text { Machare-Delgado } \\
2011[16]\end{array}$ & adults & 25 & ICU & $\begin{array}{c}\text { M-turbo, } \\
\text { Sonosite, Bothell }\end{array}$ & 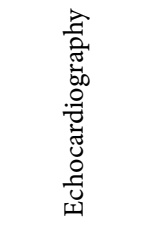 & $\begin{array}{c}\text { Mechanical } \\
\text { ventilation }(\mathrm{TV}=8.6 \\
\mathrm{mL} / \mathrm{kg})\end{array}$ & $\begin{array}{c}(\text { IVCmax - } \\
\text { IVCmin)/IVCmin }\end{array}$ & SVI $>10 \%$ & $500 \mathrm{ml}$ saline \\
\hline $\begin{array}{c}\text { Corl et al. } 2012 \\
{[17]}\end{array}$ & adults & 26 & $\mathrm{ED}$ & $\begin{array}{c}\text { M-turbo, } \\
\text { Sonosite, Bothell }\end{array}$ & 仓 & $\begin{array}{l}\text { Spontaneously } \\
\text { breathing }\end{array}$ & $\begin{array}{c}(\text { IVCmax - } \\
\text { IVCmin)/IVCmax }\end{array}$ & $\mathrm{CI}>10 \%$ & $\begin{array}{l}\text { passive leg } \\
\text { raise }\end{array}$ \\
\hline $\begin{array}{c}\text { Muller et al. } 2012 \\
{[18]}\end{array}$ & adults & 40 & ICU & $\begin{array}{c}\text { Vivid S6 } \\
\text { machine, GE }\end{array}$ & 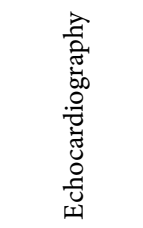 & $\begin{array}{l}\text { Spontaneously } \\
\text { breathing }\end{array}$ & $\begin{array}{c}\text { (IVCmax - } \\
\text { IVCmin)/IVCmax }\end{array}$ & $\mathrm{VTI}>15 \%$ & 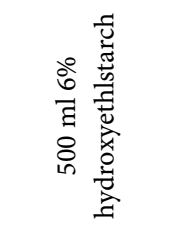 \\
\hline $\begin{array}{c}\text { Brun et al. } 2013 \\
{[19]}\end{array}$ & adults & 23 & 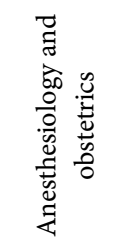 & Philips & 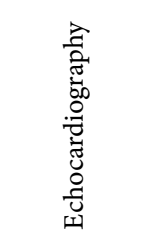 & $\begin{array}{l}\text { spontaneously } \\
\text { breathing }\end{array}$ & $\begin{array}{c}(\text { IVCmax - } \\
\text { IVCmin)/[(IVCmax } \\
+ \text { IVCmin)/2] }\end{array}$ & SVI $>15 \%$ & $\begin{array}{c}500 \mathrm{ml} \\
\text { normal saline }\end{array}$ \\
\hline
\end{tabular}




\section{Continued}

\begin{tabular}{|c|c|c|c|c|c|c|c|c|c|}
\hline $\begin{array}{c}\text { Byon HJ } 2013 \\
{[20]}\end{array}$ & children & 33 & 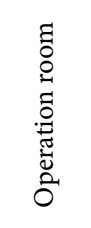 & Vivid 7, Pro, GE & 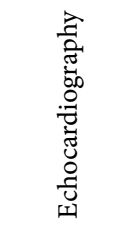 & $\begin{array}{c}\text { Mechanical } \\
\text { ventilation }(\mathrm{PEEP}= \\
\left.0 \mathrm{~cm} \mathrm{H}_{2} \mathrm{O}\right)\end{array}$ & $\begin{array}{c}(\text { IVCmax }- \\
\text { IVCmin)/[(IVCmax } \\
+ \text { IVCmin)/2] }\end{array}$ & SVI $>10 \%$ & 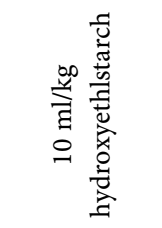 \\
\hline $\begin{array}{c}\text { Baker et al. } 2013 \\
\text { [21] }\end{array}$ & adults & 25 & ICU & Philips & 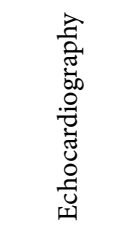 & $\begin{array}{c}\text { Mechanical } \\
\text { ventilation (TV } 6 \text { - } 8 \\
\mathrm{~mL} / \mathrm{kg} ; \text { PEEP } 5 \text { - } 8 \\
\text { cmH2O }\end{array}$ & $\begin{array}{c}\text { (IVCmax - } \\
\text { IVCmin)/IVCmin }\end{array}$ & SV $>15 \%$ & $\begin{array}{l}500 \mathrm{ml} \\
\text { colloid }\end{array}$ \\
\hline $\begin{array}{c}\text { Lanspa et al. } 2013 \\
\text { [22] }\end{array}$ & adults & 14 & ICU & Philips & 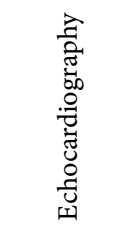 & $\begin{array}{l}\text { Spontaneously } \\
\text { breathing }\end{array}$ & $\begin{array}{c}(\text { IVCmax - } \\
\text { IVCmin)/IVCmax }\end{array}$ & $\mathrm{CI}>15 \%$ & $\begin{array}{l}10 \mathrm{ml} / \mathrm{kg} \\
\text { crystalloid }\end{array}$ \\
\hline $\begin{array}{c}\text { Charbonneau et } \\
\text { al. } 2014 \text { [23] }\end{array}$ & adults & 44 & ICU & Philips & 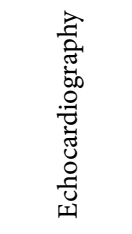 & $\begin{array}{c}\text { Mechanical } \\
\text { ventilation }(\mathrm{TV}=6.4 \\
-11.0 \mathrm{~mL} / \mathrm{kg} ; \mathrm{PEEP} \\
\left.=5-12 \mathrm{~cm} \mathrm{H} \mathrm{H}_{2} \mathrm{O}\right)\end{array}$ & $\begin{array}{c}(\mathrm{IVCmax}- \\
\text { IVCmin)/IVCmin }\end{array}$ & $\mathrm{CI}>15 \%$ & 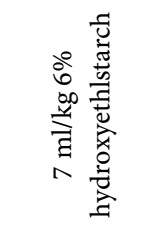 \\
\hline $\begin{array}{c}\text { de Valk et al. } 2014 \\
{[24]}\end{array}$ & 4 adults & 45 & $\mathrm{ED}$ & $\begin{array}{c}\text { Zonare, } \\
\text { Mountain View }\end{array}$ & 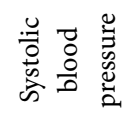 & $\begin{array}{l}\text { Spontaneously } \\
\text { breathing }\end{array}$ & $\begin{array}{c}(\text { IVCmax - } \\
\text { IVCmin)/IVCmax }\end{array}$ & $\begin{array}{c}\mathrm{SBP}>10 \\
\mathrm{mmHg}\end{array}$ & $\begin{array}{c}500 \mathrm{ml} 0.9 \% \\
\mathrm{NaCl}\end{array}$ \\
\hline $\begin{array}{c}\text { Sobczyk et al. } \\
2015 \text { [25] }\end{array}$ & adults & 50 & $\mathrm{ICU}$ & Philips & 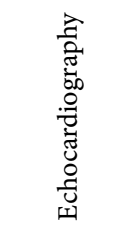 & 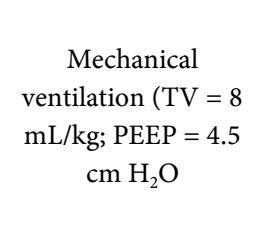 & $\begin{array}{c}\text { (IVCmax - } \\
\text { IVCmin)/IVCmin; } \\
(\text { IVCmax - } \\
\text { IVCmin)/IVCmax }\end{array}$ & $\mathrm{CO}>15 \%$ & $\begin{array}{c}2625 \pm 778 \\
\mathrm{~mL} \text { within } \\
\text { the first } 6 \\
\text { hours }\end{array}$ \\
\hline $\begin{array}{c}\text { Lujan,varas et al. } \\
2015 \text { [26] }\end{array}$ & adults & 15 & ICU & Not mention & 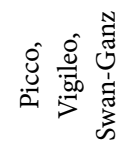 & $\begin{array}{c}\text { Mechanical } \\
\text { ventilation } \\
(\text { PEEP11.4 } \pm 3.74)\end{array}$ & $\begin{array}{c}(\text { IVCmax - } \\
\text { IVCmin)/IVCmin }\end{array}$ & $\mathrm{CO}>15 \%$ & $\begin{array}{l}\text { passive leg } \\
\text { raise }\end{array}$ \\
\hline $\begin{array}{l}\text { Airapetian et al. } \\
\quad 2015 \text { [27] }\end{array}$ & adults & 59 & ICU & Philips & 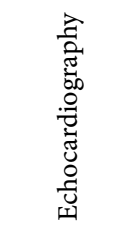 & $\begin{array}{l}\text { Spontaneously } \\
\text { breathing }\end{array}$ & $\begin{array}{c}(\text { IVCmax - } \\
\text { IVCmin)/IVCmax }\end{array}$ & $\mathrm{CO}>10 \%$ & $500 \mathrm{ml}$ saline \\
\hline $\begin{array}{c}\text { Weber et al. } 2015 \\
{[28]}\end{array}$ & children & 31 & PICU & Vivid S6; GE & 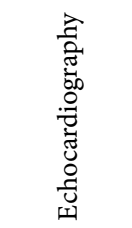 & $\begin{array}{c}\text { Mechanical } \\
\text { ventilation }(\mathrm{TV}=7.9 \\
\pm 3.8 \mathrm{~mL} / \mathrm{kg} ; \mathrm{PEEP}= \\
\left.6.8 \pm 1.8 \mathrm{~cm} \mathrm{H}_{2} \mathrm{O}\right)\end{array}$ & $\begin{array}{c}(\mathrm{IVCmax}- \\
\mathrm{IVCmin}) / \mathrm{IVCmin}\end{array}$ & SVI $>10 \%$ & 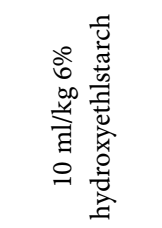 \\
\hline
\end{tabular}




\section{Continued}

\begin{tabular}{|c|c|c|c|c|c|c|c|c|c|}
\hline $\begin{array}{c}\text { Achar et al. } 2016 \\
\text { [29] }\end{array}$ & children & 42 & $\begin{array}{l}\text { Operation } \\
\text { room }\end{array}$ & $\begin{array}{l}\text { Vivid e; } \\
\text { GE }\end{array}$ & 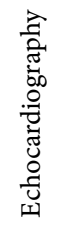 & $\begin{array}{c}\text { Mechanical } \\
\text { ventilation }(\mathrm{TV}=10 \\
\mathrm{mL} / \mathrm{kg} ; \mathrm{PEEP}=0 \mathrm{~cm} \\
\left.\mathrm{H}_{2} \mathrm{O}\right)\end{array}$ & $\begin{array}{c}(\text { IVCmax - } \\
\text { IVCmin)/IVCmin }\end{array}$ & SVI $>15 \%$ & $\begin{array}{c}10 \mathrm{ml} / \mathrm{kg} 1 \% \\
\text { dextrose } \\
\text { Ringer's } \\
\text { lactate }\end{array}$ \\
\hline $\begin{array}{c}\text { Sobczyk et al. } \\
2016[30]\end{array}$ & adults & 35 & ICU & Philips & 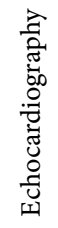 & $\begin{array}{c}\text { Mechanical } \\
\text { ventilation }(\mathrm{TV}=8 \\
\mathrm{mL} / \mathrm{kg} ; \mathrm{PEEP}=4.5 \\
\left.\mathrm{~cm} \mathrm{H}_{2} \mathrm{O}\right)\end{array}$ & $\begin{array}{c}(\text { IVCmax - } \\
\text { IVCmin)/IVCmin }\end{array}$ & $\mathrm{CO}>15 \%$ & $250 \mathrm{ml}$ saline \\
\hline $\begin{array}{c}\text { de Oliveira et } \\
\text { al.2016 [31] }\end{array}$ & adults & 20 & ICU & $\begin{array}{l}\text { Samsung } \\
\text { Medison }\end{array}$ & 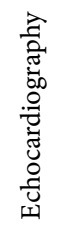 & $\begin{array}{c}\text { Mechanical } \\
\text { ventilation }(\mathrm{TV}=8 \\
\mathrm{mL} / \mathrm{kg} ; \mathrm{PEEP}=5-6 \\
\left.\mathrm{~cm} \mathrm{H}_{2} \mathrm{O}\right)\end{array}$ & $\begin{array}{c}(\text { IVCmax - } \\
\text { IVCmin)/IVCmin }\end{array}$ & $\mathrm{VTI}>15 \%$ & $\begin{array}{l}500 \\
\text { crystalloid }\end{array}$ \\
\hline
\end{tabular}

IVCmax and IVCmin = maximum and minimum diameter of inferior vena cava during a complete respiratory cycle; CI = cardiac index; CO = cardiac output; VTI = velocity-time index; SV = stroke volume; SVI = stroke volume index; SBP = systolic blood pressure; TV = tidal volume.

small, ranging from 14 to 50 patients. A total of 635 patients were included. 16 studies [12] [13] [14] [16] [17] [18] [19] [21]-[27] [30] [31] enrolled adults, and 4 studies [15] [20] [28] [29] enrolled pediatric patients. 14 studies [12] [13] [14] [15] [16] [20] [21] [23] [25] [26] [28] [29] [30] [31] enrolled patients on mechanical ventilation, and 6 studies [17] [18] [19] [22] [24] [27] enrolled spontaneously breathing patients. The formulas for the calculation of $\triangle \mathrm{IVC}$ during the respiratory cycle were different. (IVCmax - IVCmin)/IVCmin was used in 11 studies [12] [14] [16] [21] [23] [25] [26] [28] [29] [30] [31], (IVCmax - IVCmin)/IVCmax was used in 5studies [17] [18] [22] [24] [27] and (IVCmax IVCmin)/[(IVCmax + IVCmin)/2] was used in 4 studies [13] [15] [19] [20]. Interestingly, the 11 studies using the formula (IVCmax - IVCmin)/IVCmin allfocused on mechanically ventilatedpatients, and the 5 studies using the formula (IVCmax - IVCmin)/IVCmaxall focused on spontaneously breathing patients. In the 4 studies using (IVCmax - IVCmin)/[(IVCmax + IVCmin)/2] as the formula, one study focused on spontaneously breathing patients, while the other three studies focused on mechanically ventilated patients. With respect to reference standard, fluid responsiveness was defined as an increase in SV, CO or surrogate by more than $15 \%$ in 14 studies [12] [13] [14] [15] [18] [19] [21] [22] [23] [25] [26] [29] [30] [31], 10\% in 5 studies [16] [17] [20] [27] [28], and increase in SBP by more than $10 \mathrm{mmHg}$ in 1 study [24]. 16 studies [12] [13] [15] [16] [18]-[23] [25]-[31] used echocardiography to measured SV, CO or surrogate, 2 studies [14] [26] used transpulmonary thermodilution technique to measure CO, 1 study [17] used bioimpedance to measure cardiac index (CI) and the last study [24] used arterial catheter to measure SBP. VE was performed by 
crystalloids in 8 studies [16] [19] [22] [24] [27] [29] [30] [31], by colloids in 10 studies [12] [13] [14] [15] [18] [20] [21] [23] [25] [28], passive leg raise in 2 studies [17] [26]. Quality assessment according to QUADAS-2 criteria is outlined in Figure 2.

\subsection{Prediction of Fluid Responsiveness by $\Delta I V C$}

The diagnostic performance of $\triangle \mathrm{IVC}$ in each study is showed in Table 2. The

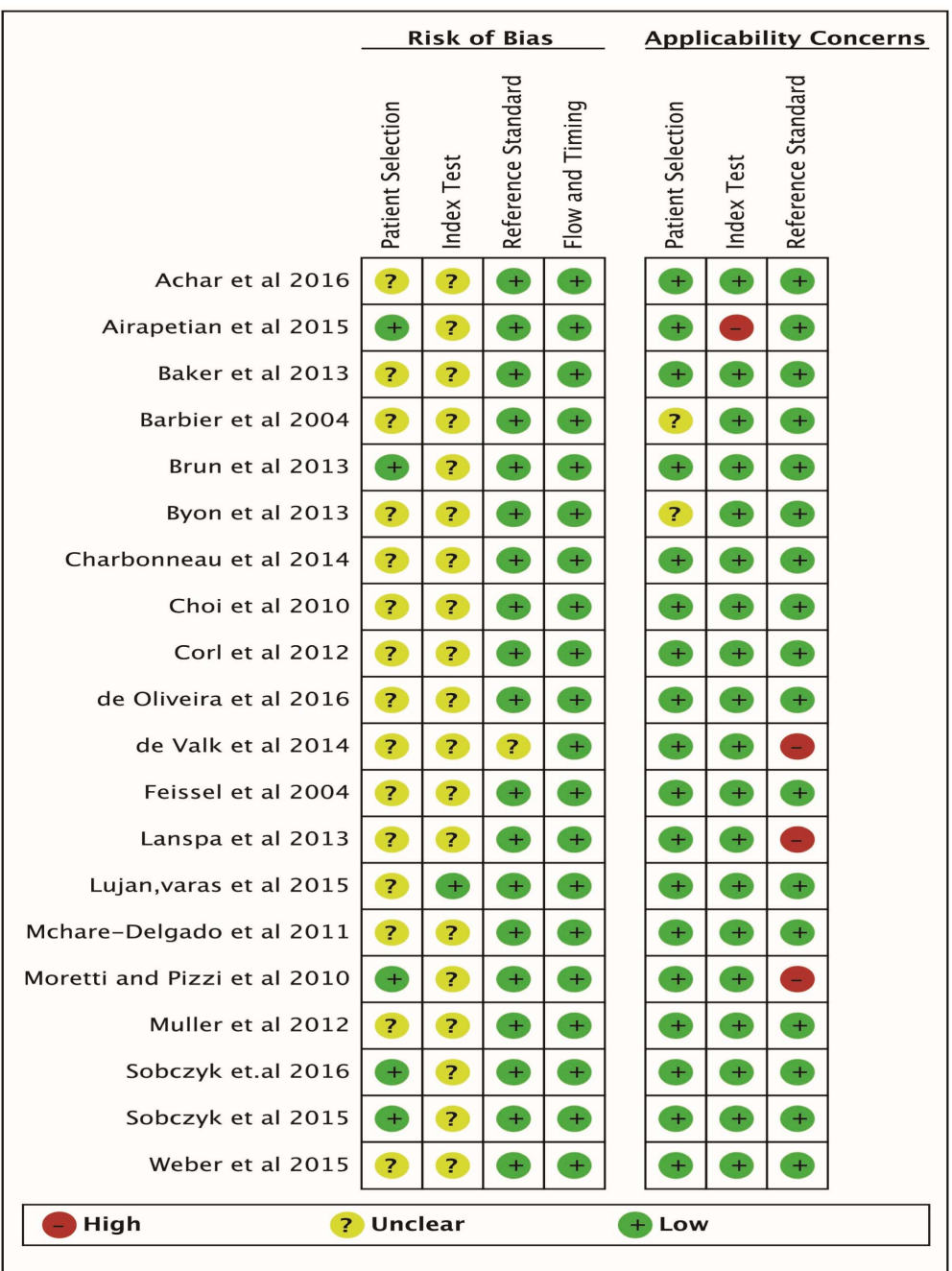

(a)

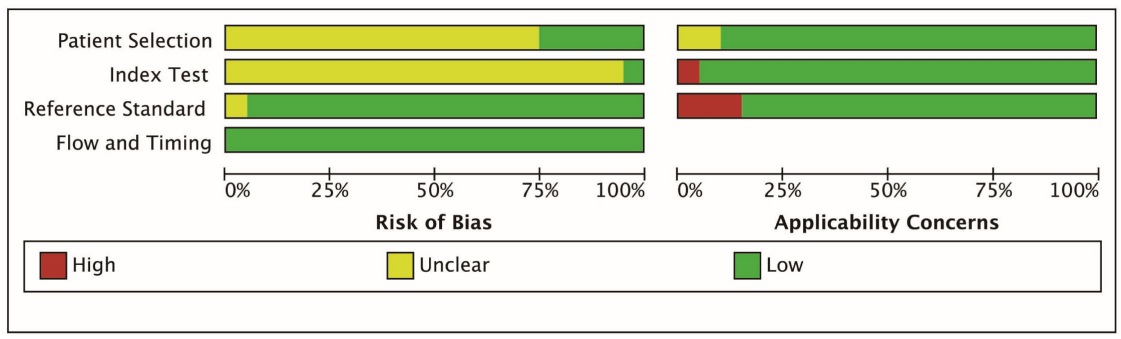

(b)

Figure 2. QUDAS-2 results and summary. 
Table 2. Sensitivity and specificity of $\triangle I V C$ in predicting fluid responsiveness.

\begin{tabular}{|c|c|c|c|c|c|c|c|c|}
\hline Study & TP & FP & FN & TN & $\begin{array}{l}\text { Cutoff } \\
\text { value }\end{array}$ & $\begin{array}{c}\text { Sensitivity } \\
\text { (\%) }\end{array}$ & $\begin{array}{l}\text { Specificity } \\
(\%)\end{array}$ & $\begin{array}{l}\text { AUROC } \\
(95 \% \text { CI })\end{array}$ \\
\hline Barbier et al. 2004 & 9 & 1 & 1 & 9 & $18 \%$ & 90.00 & $90 \%$ & $0.91(0.84,0.98)$ \\
\hline Feissel et al. 2004 & 14 & 1 & 2 & 22 & $12 \%$ & - & - & - \\
\hline $\begin{array}{l}\text { Moretti and Pizzi } \\
2010\end{array}$ & 12 & 0 & 5 & 12 & $16 \%$ & $70.59 \%$ & $100 \%$ & $0.902(0.733,0.979)$ \\
\hline Deok et al. 2010 & - & - & - & - & - & - & - & $0.85(0.69,1.00)$ \\
\hline $\begin{array}{c}\text { Machare-Delgado } \\
2011\end{array}$ & 8 & 8 & 0 & 9 & $12 \%$ & 100.00 & $53 \%$ & $0.81(0.64,0.99)$ \\
\hline Corl et al. 2012 & - & - & - & - & - & - & - & $0.46(0.21,0.71)$ \\
\hline Muller et al. 2012 & 14 & 4 & 6 & 16 & $40 \%$ & 70 & $80 \%$ & $0.77(0.60,0.88)$ \\
\hline Brun et al. 2013 & - & - & - & - & - & - & - & $0.57(0.32,0.82)$ \\
\hline Byon HJ 2013 & - & - & - & - & - & - & - & $0.369(0.156,0.582)$ \\
\hline Baker et al. 2013 & - & - & - & - & - & - & - & $0.46(0.22-0.69)$ \\
\hline Lanspa et al. 2013 & 5 & 3 & 0 & 6 & $15 \%$ & 100 & $66.66 \%$ & $0.83(0.58-1.0)$ \\
\hline $\begin{array}{c}\text { Charbonneau et al. } \\
2014\end{array}$ & 10 & 7 & 16 & 11 & $21 \%$ & 38 & $61 \%$ & $0.43(0.25,0.61)$ \\
\hline de Valk et al. 2014 & 10 & 11 & 2 & 22 & 36.5 & 83 & $67 \%$ & 0.741 \\
\hline Sobczyk et al. 2015 & - & - & - & - & - & - & - & - \\
\hline $\begin{array}{l}\text { Lujan, varas et al. } \\
2015\end{array}$ & 2 & 2 & 1 & 10 & $18 \%$ & - & - & - \\
\hline $\begin{array}{l}\text { Airapetian et al. } \\
2015\end{array}$ & 9 & 1 & 20 & 29 & $42 \%$ & 31 & $97 \%$ & $\begin{array}{c}0.62 \pm 0.07 \\
(0.49-0.74)\end{array}$ \\
\hline Weber et al. 2015 & - & - & - & - & - & - & - & $0.502(0.29,0.71)$ \\
\hline Achar et al. 2016 & 22 & 2 & 2 & 16 & $23.5 \%$ & 91 & $89 \%$ & 0.94 \\
\hline Sobczyk et al. 2016 & 20 & 3 & 4 & 8 & $18 \%-$ & $82.35 \%-$ & $72.72 \%-$ & 0.739 \\
\hline $\begin{array}{c}\text { de Oliveira et al. } \\
2016\end{array}$ & 6 & 0 & 3 & 11 & $16 \%$ & 66.67 & $100 \%$ & $\begin{array}{l}0.84 \pm 0.10 \\
(0.63-1.0)\end{array}$ \\
\hline
\end{tabular}

$\mathrm{TP}=$ true positive; $\mathrm{FP}=$ false positive; $\mathrm{FN}=$ false negative; $\mathrm{TN}=$ true negative; $\mathrm{AUROC}=$ area under the receiver operating characteristic curve; $\mathrm{CI}=$ confidence interval.

sensitivity and specificity was reported in 14 studies [12] [13] [14] [16] [18] [19] [22] [23] [24] [26] [27] [29] [30] [31]. The pooled sensitivity, specificity and DOR was 0.68 (0.62 - 0.75), $0.80(0.75$ - 0.85) and 14.2 (6.0 - 33.6), respectively. (Table 2, Figure 3). The area under the corresponding ROC curve was reported in 17 studies [12] [14]-[24] [27] [28] [29] [30] [31]. In 9 studies [12] [14] [15] [16] [18] [22] [24] [29] [31], the AUROC of $\triangle \mathrm{IVC}$ were more than 0.7, and in the other 8 studies [17] [19] [20] [21] [23] [27] [28] [30], $\Delta$ IVC showed low diagnostic value. The pooled AUROC was 0.86 (0.78 - 0.93) (Table 2, Figure 4). The threshold of $\triangle \mathrm{IVC}$ was reported in 13 studies [12] [13] [14] [16] [18] [22] [23] [24] [26] [27] [29] [30] [31], the values varied across studies, ranging from $12 \%$ to $42 \%$ (Table 2 ). 


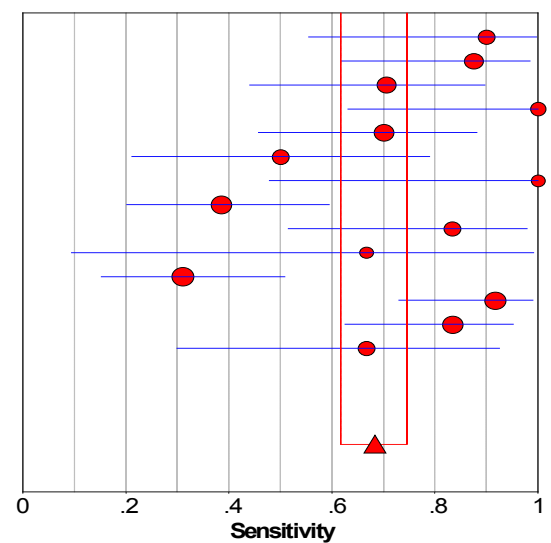

Barbier et.al

Feissel et.al

Moretti and Pizzi

Machare-Delgado
Muller et.al

Brun et.al

Lanspa et.al

Charbonneau et.al

de Valk et.al

Lujan.varas et.al

Airapetian et.al

Acher et.al

Sobczykle et.a

de Oliveira et.a

Chi-square $=56.05 ; \mathrm{df}=13(\mathrm{p}=0.0000)$

Inconsistency (I-square) $=76.8 \%$

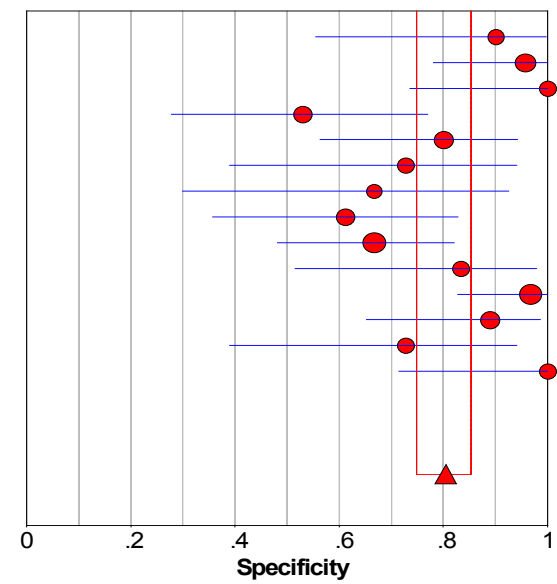

Barbier et.a

Feissel et.a

Moretti and Pizzi

Machare-Delgado

Muller et.al

Brun et.al

Lanspa et.al

Charbonneau et.al

de Valk et.al

Lujan.varas et.al

Airapetian et.al

Acher et.al

Sobczykle et.al

de Oliveira et.al

Pooled Specificity $=0.80(0.75$ to 0.85$)$

Chi-square $=38.69 \cdot \mathrm{df}=13(\mathrm{p}=0.0002)$

Inconsistency (I-square) $=66.4 \%$

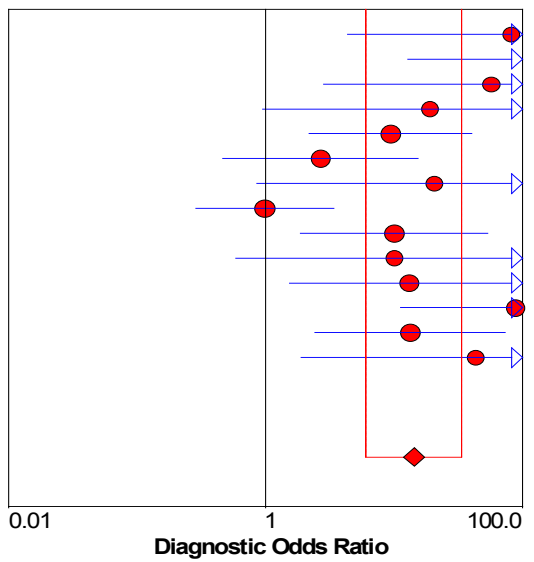

Barbier et.al

Feissel et.al

Moretti and Pizzi

Machare-Delgado

Muller et.al

Brun et.al

Lanspa et.al

Charbonneau et.al

de Valk et.al

Lujan.varas et.al

Airapetian et.al

Acher et.al

Sobczykle et.a

de Oliveira et.al

Random Effects Model

Pooled Diagnostic Odds Ratio $=14.24$ (6.04 to 33.61)

Cochran- $Q=29.35 ; d f=13(p=0.0058)$

Inconsistency (I-square) $=55.7 \%$

Tau-squared $=1.3803$
Diagnostic OR (95\% Cl)

$81.00 \quad(4.36-1,504.48)$

$154.00 \quad(12.74-1,861.59)$

$56.82(2.83-1,140.47)$

$19.00 \quad(0.95-381.07)$

$9.33(2.18-39.96)$

$2.67(0.47-15.25)$

$20.43(0.86-487.97)$

$0.98 \quad(0.29-3.37)$

$10.00(1.86-53.76)$

$10.00 \quad(0.58-171.20)$

$13.05 \quad(1.53-111.26)$

$88.00 \quad(11.18-692.56)$

$13.33(2.42-73.48)$

$42.71(1.89-962.86)$

Figure 3. Pooled diagnostic accuracy of $\triangle \mathrm{IVC}$ in whole studies.

\subsection{Subgroup Analysis and Investigation of Heterogeneity}

The Spearman correlation coefficient between sensitivity and specificity was 0.323 ( $\mathrm{p}=0.260$ ), indicating no threshold effect. The heterogeneity Chi-squared was $56 \%$ for sensitivity and $39 \%$ for specificity. The $P$ statistics was $77 \%$ for sensitivity, $66 \%$ for specificity.

Meta-regression shows none of the covariates included were the significant source of heterogeneity. However, the comparison between studies with mechanical ventilation versus studies with spontaneously breathing, and between 


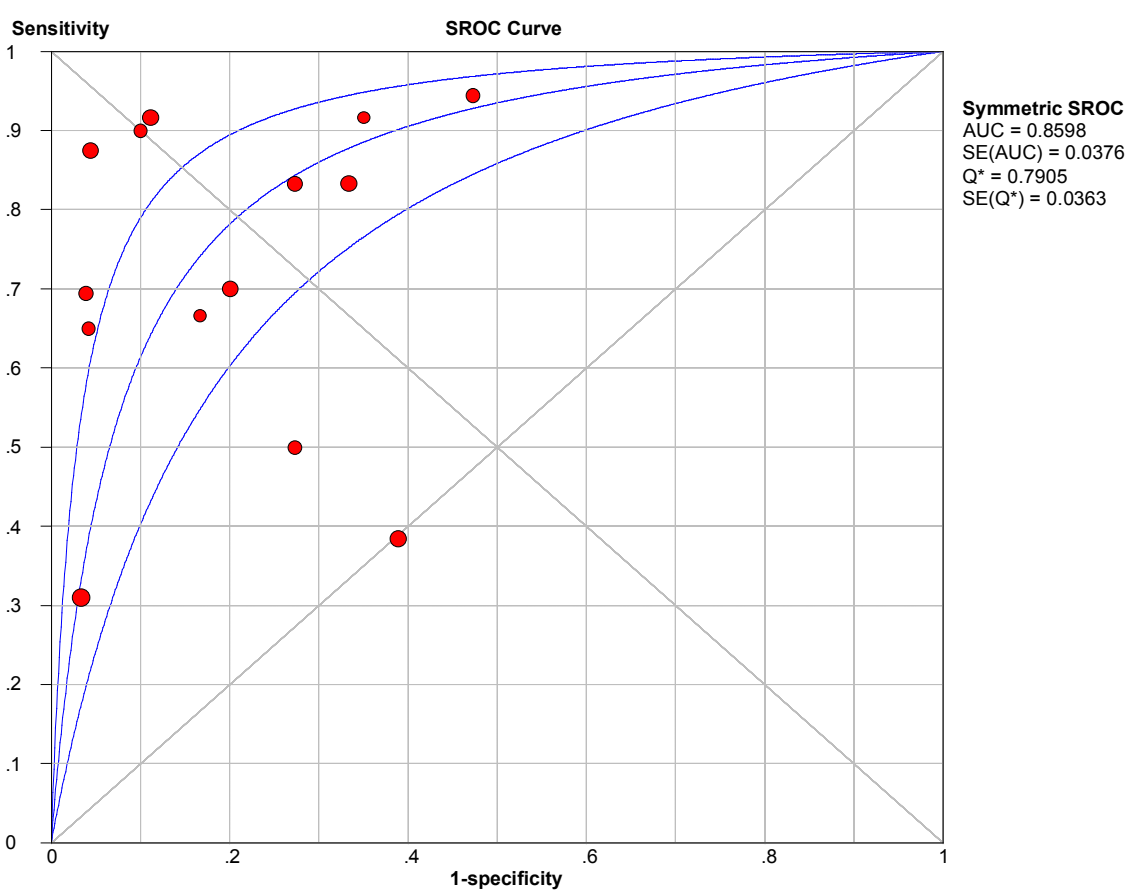

Figure 4. Summary receiver operating characteristics curve of $\triangle I V C$ in whole studies.

studies with different devices and formulas for the calculation of $\triangle \mathrm{IVC}$ had influence on sensitivity and specificity. Diagnostically, $\triangle \mathrm{IVC}$ performed better in patients on mechanical ventilation than in spontaneously breathing patients with higher sensitivity ( 0.75 vs. 0.56$)$, specificity ( 0.82 vs. 0.78$)$, DOR (22.9 vs. 7.9), and AUROC (0.9 vs.0.8) (Table 3). In addition, 9 studies [12] [13] [14] [16] [23] [26] [29] [30] [31] with mechanical ventilation reported the threshold ranging from $12 \%$ to $23.5 \%$, the average was $17 \% \pm 4 \%$; the average of the other 4 studies [18] [22] [24] [27] with spontaneously breathing was $33 \% \pm 12 \%$.

\section{Discussion}

This meta-analysis including 20 studies with a combined total of 635 patients concluded that ICU staff must be cautious of using $\triangle \mathrm{IVC}$, which was not so excellent to predict fluid responsiveness with pooled sensitivity $(0.68)$ and specificity $(0.80)$.In patients on mechanical ventilation, $\triangle \mathrm{IVC}$ could predict fluid responsiveness moderately with acceptable pooled sensitivity (0.75) and specificity (0.82). The pooled AUROC was $0.90(0.80-0.99)$ and the average of threshold was $\triangle \mathrm{IVC} \geq 17 \% \pm 4 \%$. However, in spontaneously breathing patients, $\triangle \mathrm{IVC}$ predict fluid responsiveness with poor sensitivity $(0.56)$ and acceptable specificity (0.78).

Point-of-care ultrasonography is a reliable monitoring technique and is becoming increasingly popular in the ICU. The IVC diameter is easily examined from a subcostal view in a longitudinal section, varying during the respiratory cycle due to the changes in intrathoracic pressure during inspiration and expiration. This variation is expressed as the $\triangle \mathrm{IVC}$. Recent years, $\Delta \mathrm{VC}$ has been developed to 
Table 3. Pooled diagnostic accuracy of $\triangle \mathrm{IVC}$ in whole and subgroup studies.

\begin{tabular}{|c|c|c|c|c|c|c|c|}
\hline Setting & $\begin{array}{c}\text { Total number } \\
\text { of studies }\end{array}$ & $\begin{array}{l}\text { Sensitivity } \\
(95 \% \text { CI })\end{array}$ & $\begin{array}{l}\text { Specificity } \\
(95 \% \mathrm{CI})\end{array}$ & $\begin{array}{l}\text { Diagnostic odds } \\
\text { ratio }(95 \% \mathrm{CI})\end{array}$ & $\begin{array}{l}\text { Positive likelihood } \\
\text { ratio }(95 \% \mathrm{CI})\end{array}$ & $\begin{array}{l}\text { Negative likelihood } \\
\text { ratio }(95 \% \mathrm{CI})\end{array}$ & AUROC \\
\hline Overall & 14 & $0.68(0.62-0.75)$ & $0.80(0.75-0.85)$ & $14.2(6.0-33.6)$ & $3.3(2.1-5.1)$ & $0.34(0.21-0.54)$ & $0.86(0.78-0.93)$ \\
\hline $\begin{array}{l}\text { Mechanical } \\
\text { ventilation }\end{array}$ & 9 & $0.75(0.67-0.82)$ & $0.82(0.74-0.88)$ & $22.9(5.6-93.4)$ & $4.3(2.0-9.4)$ & $0.27(0.13-0.54)$ & $0.90(0.80-0.99)$ \\
\hline $\begin{array}{l}\text { Spontaneous } \\
\text { breathing }\end{array}$ & 5 & $0.56(0.45-0.68)$ & $0.78(0.70-0.86)$ & $7.9(3.5-18.1)$ & $2.7(1.8-4.0)$ & $0.50(0.29-0.86)$ & $0.80(0.71-0.89)$ \\
\hline
\end{tabular}

accurately predict fluid responsiveness in clinical practice. The consensus on circulatory shock and hemodynamic monitoring published by task force of the European Society of Intensive Care Medicine in 2014 recommended that $\triangle I V C$ as dynamic variables were available to predict fluid responsiveness [32].

To our knowledge, in 2014, Zhang and co-workers performed a systematic review and meta-analysis that included eight studies investigating the diagnostic performance of $\triangle \mathrm{IVC}$ [9]. They concluded that $\triangle \mathrm{IVC}$ is of great value in predicting fluid responsiveness, particularly in patients on mechanical ventilation compared to spontaneously breathing patients. However, since this meta-analysis, additional studies [19] [20] [21] [23] [25] [26] [27] [28] have been published, reporting $\triangle \mathrm{IVC}$ would not be reliable in spontaneously breathing patients. In addition, G. Via et al. [33] have suggested ten situations where $\triangle \mathrm{IVC}$ may fail to accurately predict fluid responsiveness. Furthermore, the threshold of $\triangle \mathrm{IVC}$ varied widely, causing confusion of ICU staff to use it in clinical practice. Finally, the meta-analysis of Zhang et al. included only one study [18] investigating spontaneously breathing patients and four studies [12] [13] [14] [16] on mechanical ventilation with complete data. All these arguments justified an updated meta-analysis.

Our meta-analysis is inconsistent with the meta-analysis performed by Zhang et al. and concluded that ICU staff must be cautious of using $\triangle \mathrm{IVC}$ to test fluid responsiveness. Based on the results from a large number of patients, we found that $\triangle \mathrm{IVC}$ was not so excellent to predict fluid responsiveness with poor sensitivity (0.68) and acceptable specificity (0.80). The pooled AUROC was 0.86 but not close to each other. In addition, the threshold values for $\triangle \mathrm{IVC}$ varied across studies, ranging from $12 \%$ to $42 \%$, which reinforce our conclusion.

In subgroup analysis, our study indicated that in patients on mechanical ventilation, $\triangle \mathrm{IVC}$ predict fluid responsiveness with acceptable pooled sensitivity (0.75) and specificity (0.82), which are less accurate than meta-analysis performed by Zhang et al., however. This is likely due to high PEEP and/or low tidal volume invalidating the diagnostic performance of $\triangle \mathrm{IVC}$. High PEEP has been demonstrated to elevate right atrial pressure (RAP) and IVC pressure, while simultaneously reducing venous return, introducing an increase IVC size and false negative of $\triangle \mathrm{IVC}$ [34]. Furthermore, the low tidal volumes less than $8 \mathrm{ml} / \mathrm{kg}$ will cause smaller variations in intrathoratic blood volume, resulting in smaller $\triangle I V C$ theoretically, irrespective of volume status. Charbonneau et al. [23] sug- 
gested that $\triangle \mathrm{IVC}$ predicted fluid responsiveness with low sensitivity (38\%), and Baker et al. [21] demonstrated that $\triangle \mathrm{IVC}$ was an inaccuracy predictor with low AUROC (0.46). The ventilation of these two studies was High PEEP $>5 \mathrm{~cm} \mathrm{H}_{2} \mathrm{O}$ and low tidal volumes $<8 \mathrm{ml} / \mathrm{kg}$. However, these two studies [21] [23] were published after the meta-analysis performed by Zhang et al. In addition, our study indicated that in spontaneously breathing patients, $\triangle \mathrm{IVC}$ predict fluid responsiveness with poor sensitivity (0.56) and acceptable specificity (0.78). The pooled AUROC was $0.80(0.71-0.89)$. This is probably because of varying breath, meaning that the amplitude of intrathoracic pressure swings and size of tidal volumes are hard to quantify in spontaneously breathing patients. Study in healthy volunteers [35] shows deeper the breathing is, the larger diaphragmatic motion and $\triangle \mathrm{IVC}$ are, regardless of volume status. This indicates that shallow breaths may minify $\triangle \mathrm{IVC}$ and reduce its sensitivity, while inspiratory efforts may magnify $\triangle \mathrm{IVC}$ and reduce its specificity [18]. Even if in patients on ventilation, $\triangle I V C$ is not a valid measure when patients made an inspiratory effort [36].

An important point that must be paid more attention to is the formula of calculation of $\triangle \mathrm{IVC} . \triangle \mathrm{IVC}$ is usually expressed as the difference between expiratory IVC diameter and inspiratory IVC diameter divided by the expiratory IVC diameter, multiplied by $100 \%$. However, in spontaneous respiration or mechanical ventilation, the changes of IVC diameter are opposite because of opposite changes of intrathoracic pressure during inspiration. In patients on mechanical ventilation, $\triangle \mathrm{IVC}$ is calculated by (IVCmax - IVCmin)/IVCmin defined as IVC distensibility index ( $\triangle \mathrm{dIVC})$, while in spontaneously breathing patients, it is calculated by (IVCmax - IVCmin)/IVCmax defined as IVC collapsibility index $(\triangle \mathrm{cIVC})$. In our meta-analysis, the best threshold of $\triangle \mathrm{IVC}$ in patients on mechanical ventilation was $\Delta \mathrm{dIVC} \geq 17 \% \pm 4 \%$, compared to $\Delta \mathrm{cIVC} \geq 33 \% \pm 12 \%$ in spontaneously breathing patients. Nowadays, the clinical use of $\triangle \mathrm{IVC}$ is in chaos regardless of its physiology, leading to misjudgment, which need to be more accurate define and recognition.

There are some limitations that should be noted for interpreting the results. First, the heterogeneity of the included studies existed with respect to patient population, respiratory pattern, calculation formula, definition of index test and fluid responsiveness. Nevertheless, no threshold effect was detected. Furthermore, both the subgroup analyses and meta-regression were opposed to the influence of heterogeneity on the results. Second, although we performed subgroup analysis, the number of studies and sample size in each subgroup was small, the conclusion needs to be validated in future trials. Third, we did not include studies not in English, non-full-text and unpublished studies, which may increase the risk of reporting bias.

\section{Conclusion}

In conclusion, our meta-analysis indicated that $\triangle \mathrm{IVC}$ is not an excellent predictor of fluid responsiveness in patients with acute circulatory failure. The pre- 
dicting ability of $\triangle \mathrm{IVC}$ was moderate in patients on mechanical ventilation, while it was poor in spontaneously breathing patients. Thus, intensivist must be cautious of using $\triangle \mathrm{IVC}$.

\section{Conflicts of Interest}

The authors declare no conflicts of interest regarding the publication of this paper.

\section{References}

[1] Lakhal, K., Ehrmann, S., Perrotin, D., Wolff, M. and Boulain, T. (2013) Fluid Challenge: Tracking Changes in Cardiac Output with Blood Pressure Monitoring (Invasive or Non-Invasive). Intensive Care Medicine, 39, 1953-1962. https://doi.org/10.1007/s00134-013-3086-6

[2] Marik, P.E., Monnet, X. and Teboul, J.L. (2011) Hemodynamic Parameters to Guide Fluid Therapy. Annals of Intensive Care, $1,1$. https://doi.org/10.1186/2110-5820-1-1

[3] Michard, F. and Teboul, J.L. (2002) Predicting Fluid Responsiveness in ICU Patients: A Critical Analysis of the Evidence. Chest, 121, 2000-2008. https://doi.org/10.1378/chest.121.6.2000

[4] Osman, D., Ridel, C., Ray, P., Monnet, X., Anguel, N., Richard, C. and Teboul, J.L. (2007) Cardiac Filling Pressures Are Not Appropriate to Predict Hemodynamic Response to Volume Challenge. Critical Care Medicine, 35, 64-68. https://doi.org/10.1097/01.CCM.0000249851.94101.4F

[5] Slama, M., Masson, H., Teboul, J.L., Arnout, M.L., Susic, D., Frohlich, E. and Andrejak, M. (2002) Respiratory Variations of Aortic VTI: A New Index of Hypovolemia and Fluid Responsiveness. American Journal of Physiology Heart and Circulatory Physiology, 283, H1729-1733. https://doi.org/10.1152/ajpheart.00308.2002

[6] Feissel, M., Michard, F., Mangin, I., Ruyer, O., Faller, J.P. and Teboul, J.L. (2001) Respiratory Changes in Aortic Blood Velocity as an Indicator of Fluid Responsiveness in Ventilated Patients with Septic Shock. Chest, 119, 867-873.

https://doi.org/10.1378/chest.119.3.867

[7] Monnet, X., Bleibtreu, A., Ferre, A., Dres, M., Gharbi, R., Richard, C. and Teboul, J.L. (2012) Passive Leg-Raising and End-Expiratory Occlusion Tests Perform Better than Pulse Pressure Variation in Patients with Low Respiratory System Compliance. Critical Care Medicine, 40, 152-157. https://doi.org/10.1097/CCM.0b013e31822f08d7

[8] Matamis, D., Soilemezi, E., Tsagourias, M., Akoumianaki, E., Dimassi, S., Boroli, F., Richard, J.C. and Brochard, L. (2013) Sonographic Evaluation of the Diaphragm in Critically Ill Patients. Technique and Clinical Applications. Intensive Care Medicine, 39, 801-810. https://doi.org/10.1007/s00134-013-2823-1

[9] Zhang, Z., Xu, X., Ye, S. and Xu, L. (2014) Ultrasonographic Measurement of the Respiratory Variation in the Inferior Vena Cava Diameter Is Predictive of Fluid Responsiveness in Critically Ill Patients: Systematic Review and Meta-Analysis. UItrasound in Medicine \& Biology, 40, 845-853.

https://doi.org/10.1016/j.ultrasmedbio.2013.12.010

[10] Schardt, C., Adams, M.B., Owens, T., Keitz, S. and Fontelo, P. (2007) Utilization of the PICO Framework to Improve Searching PubMed for Clinical Questions. BMC Medical Informatics and Decision Making, 7, 16. 
https://doi.org/10.1186/1472-6947-7-16

[11] Whiting, P.F., Rutjes, A.W., Westwood, M.E., Mallett, S., Deeks, J.J., Reitsma, J.B., Leeflang, M.M., Sterne, J.A. and Bossuyt, P.M. (2011) QUADAS-2: A Revised Tool for the Quality Assessment of Diagnostic Accuracy Studies. Annals of Internal Medicine, 155, 529-536. https://doi.org/10.7326/0003-4819-155-8-201110180-00009

[12] Barbier, C., Loubieres, Y., Schmit, C., Hayon, J., Ricome, J.L., Jardin, F. and Vieillard-Baron, A. (2004) Respiratory Changes in Inferior Vena Cava Diameter Are Helpful in Predicting Fluid Responsiveness in Ventilated Septic Patients. Intensive Care Medicine, 30, 1740-1746. https://doi.org/10.1007/s00134-004-2259-8

[13] Feissel, M., Michard, F., Faller, J.P. and Teboul, J.L. (2004) The Respiratory Variation in Inferior Vena Cava Diameter as a Guide to Fluid Therapy. Intensive Care Medicine, 30, 1834-1837. https://doi.org/10.1007/s00134-004-2233-5

[14] Moretti, R. and Pizzi, B. (2010) Inferior Vena Cava Distensibility as a Predictor of Fluid Responsiveness in Patients with Subarachnoid Hemorrhage. Neurocritical Care, 13, 3-9. https://doi.org/10.1007/s12028-010-9356-Z

[15] Choi, D.Y., Kwak, H.J., Park, H.Y., Kim, Y.B., Choi, C.H. and Lee, J.Y. (2010) Respiratory Variation in Aortic Blood Flow Velocity as a Predictor of Fluid Responsiveness in Children after Repair of Ventricular Septal Defect. Pediatric Cardiology, 31, 1166-1170. https://doi.org/10.1007/s00246-010-9776-8

[16] Machare-Delgado, E., Decaro, M. and Marik, P.E. (2011) Inferior Vena Cava Variation Compared to Pulse Contour Analysis as Predictors of Fluid Responsiveness: A Prospective Cohort Study. Journal of Intensive Care Medicine, 26, 116-124. https://doi.org/10.1177/0885066610384192

[17] Corl, K., Napoli, A.M. and Gardiner, F. (2012) Bedside Sonographic Measurement of the Inferior Vena Cava Caval Index Is a Poor Predictor of Fluid Responsiveness in Emergency Department Patients. Emergency Medicine Australasia, 24, 534-539. https://doi.org/10.1111/j.1742-6723.2012.01596.x

[18] Muller, L., Bobbia, X., Toumi, M., Louart, G., Molinari, N., Ragonnet, B., Quintard, H., Leone, M., Zoric, L. and Lefrant, J.Y. (2012) Respiratory Variations of Inferior Vena Cava Diameter to Predict Fluid Responsiveness in Spontaneously Breathing Patients with Acute Circulatory Failure: Need for a Cautious Use. Critical Care, 16, R188. https://doi.org/10.1186/cc11672

[19] Brun, C., Zieleskiewicz, L., Textoris, J., Muller, L., Bellefleur, J.P., Antonini, F., Tourret, M., Ortega, D., Vellin, A., Lefrant, J.Y., Boubli, L., Bretelle, F., Martin, C. and Leone, M. (2013) Prediction of Fluid Responsiveness in Severe Preeclamptic Patients with Oliguria. Intensive Care Medicine, 39, 593-600. https://doi.org/10.1007/s00134-012-2770-2

[20] Byon, H.J., Lim, C.W., Lee, J.H., Park, Y.H., Kim, H.S., Kim, C.S. and Kim, J.T. (2013) Prediction of Fluid Responsiveness in Mechanically Ventilated Children Undergoing Neurosurgery. British Journal of Anaesthesia, 110, 586-591. https://doi.org/10.1093/bja/aes467

[21] Baker, A.K., Partridge, R.J., Litton, E. and Ho, K.M. (2013) Assessment of the Plethysmographic Variability Index as a Predictor of Fluid Responsiveness in Critically Ill Patients: A Pilot Study. Anaesthesia and Intensive Care, 41, 736-741.

[22] Lanspa, M.J., Grissom, C.K., Hirshberg, E.L., Jones, J.P. and Brown, S.M. (2013) Applying Dynamic Parameters to Predict Hemodynamic Response to Volume Expansion in Spontaneously Breathing Patients with Septic Shock. Shock, 39, 155-160. https://doi.org/10.1097/SHK.0b013e31827f1c6a

[23] Charbonneau, H., Riu, B., Faron, M., Mari, A., Kurrek, M.M., Ruiz, J., Geeraerts, T., 
Fourcade, O., Genestal, M. and Silva, S. (2014) Predicting Preload Responsiveness Using Simultaneous Recordings of Inferior and Superior Vena Cavae Diameters. Critical Care, 18, 473. https://doi.org/10.1186/s13054-014-0473-5

[24] de Valk, S., Olgers, T.J., Holman, M., Ismael, F., Ligtenberg, J.J. and Ter Maaten, J.C. (2014) The Caval Index: An Adequate Non-Invasive Ultrasound Parameter to Predict Fluid Responsiveness in the Emergency Department? BMC Anesthesiology, 14, 114. https://doi.org/10.1186/1471-2253-14-114

[25] Sobczyk, D., Nycz, K. and Andruszkiewicz, P. (2015) Bedside Ultrasonographic Measurement of the Inferior Vena Cava Fails to Predict Fluid Responsiveness in the First 6 Hours after Cardiac Surgery: A Prospective Case Series Observational Study. Journal of Cardiothoracic and Vascular Anesthesia, 29, 663-669. https://doi.org/10.1053/j.jvca.2014.08.015

[26] Lujan Varas, J., Martinez Díaz, C., Blancas, R., Martinez Gonzalez, O., Llorente Ruiz, B., Molina Montero, R., Arenillas Juanas, C., Pardo, A., Alcázar Sánchez-Elvira, L. and Cambronero Galache, J.A. (2015) Inferior Vena Cava Distensibility Index Predicting Fluid Responsiveness in Ventilated Patients. Intensive Care Medicine Experimental, 3, A600. https://doi.org/10.1186/2197-425X-3-S1-A600

[27] Airapetian, N., Maizel, J., Alyamani, O., Mahjoub, Y., Lorne, E., Levrard, M., Ammenouche, N., Seydi, A., Tinturier, F., Lobjoie, E., Dupont, H. and Slama, M. (2015) Does Inferior Vena Cava Respiratory Variability Predict Fluid Responsiveness in Spontaneously Breathing Patients? Critical Care, 19, 400.

https://doi.org/10.1186/s13054-015-1100-9

[28] Weber, T., Wagner, T., Neumann, K. and Deusch, E. (2015) Low Predictability of Three Different Noninvasive Methods to Determine Fluid Responsiveness in Critically Ill Children. Pediatric Critical Care Medicine, 16, e89-e94. https://doi.org/10.1097/PCC.0000000000000364

[29] Achar, S.K., Sagar, M.S., Shetty, R., Kini, G., Samanth, J., Nayak, C., Madhu, V. and Shetty, T. (2016) Respiratory Variation in Aortic Flow Peak Velocity and Inferior Vena Cava Distensibility as Indices of Fluid Responsiveness in Anaesthetised and Mechanically Ventilated Children. Indian Journal of Anaesthesia, 60, 121-126. https://doi.org/10.4103/0019-5049.176285

[30] Sobczyk, D., Nycz, K., Andruszkiewicz, P., Wierzbicki, K. and Stapor, M. (2016) Ultrasonographic Caval Indices Do Not Significantly Contribute to Predicting Fluid Responsiveness Immediately after Coronary Artery Bypass Grafting When Compared to Passive Leg Raising. Cardiovascular Ultrasound, 14, 23.

https://doi.org/10.1186/s12947-016-0065-4

[31] de Oliveira, O.H., Freitas, F.G., Ladeira, R.T., Fischer, C.H., Bafi, A.T., Azevedo, L.C. and Machado, F.R. (2016) Comparison between Respiratory Changes in the Inferior Vena Cava Diameter and Pulse Pressure Variation to Predict Fluid Responsiveness in Postoperative Patients. Journal of Critical Care, 34, 46-49. https://doi.org/10.1016/j.jcrc.2016.03.017

[32] Cecconi, M., De Backer, D., Antonelli, M., Beale, R., Bakker, J., Hofer, C., Jaeschke, R., Mebazaa, A., Pinsky, M.R., Teboul, J.L., Vincent, J.L. and Rhodes, A. (2014) Consensus on Circulatory Shock and Hemodynamic Monitoring. Task Force of the European Society of Intensive Care Medicine. Intensive Care Medicine, 40, 1795-1815. https://doi.org/10.1007/s00134-014-3525-Z

[33] Via, G., Tavazzi, G. and Price, S. (2016) Ten Situations Where Inferior Vena Cava Ultrasound May Fail to Accurately Predict Fluid Responsiveness: A Physiologically Based Point of View. Intensive Care Medicine, 42, 1164-1167. https://doi.org/10.1007/s00134-016-4357-9 
[34] Jellinek, H., Krenn, H., Oczenski, W., Veit, F., Schwarz, S. and Fitzgerald, R.D. (2000) Influence of Positive Airway Pressure on the Pressure Gradient for Venous Return in Humans. Journal of Applied Physiology, 88, 926-932. https://doi.org/10.1152/jappl.2000.88.3.926

[35] Gignon, L., Roger, C., Bastide, S., Alonso, S., Zieleskiewicz, L., Quintard, H., Zoric, L., Bobbia, X., Raux, M., Leone, M., Lefrant, J.Y. and Muller, L. (2016) Influence of Diaphragmatic Motion on Inferior Vena Cava Diameter Respiratory Variations in Healthy Volunteers. Anesthesiology, 124, 1338-1346.

https://doi.org/10.1097/ALN.0000000000001096

[36] Juhl-Olsen, P., Frederiksen, C.A. and Sloth, E. (2012) Ultrasound Assessment of Inferior Vena Cava Collapsibility Is Not a Valid Measure of Preload Changes during Triggered Positive Pressure Ventilation: A Controlled Cross-Over Study. Ultraschall in der Medizin, 33, 152-159. https://doi.org/10.1055/s-0031-1281832 